\title{
High-flux focusable color-tunable and efficient white-light-emitting diode light engine
} for stage lighting

Chakrabarti, Maumita; Pedersen, Henrik Chresten; Petersen, Paul Michael; Poulsen, Christian; Poulsen, Peter Behrensdorff; Dam-Hansen, Carsten

Published in:

Optical Engineering

Link to article, DOI:

10.1117/1.OE.55.8.085101

Publication date:

2016

Document Version

Publisher's PDF, also known as Version of record

Link back to DTU Orbit

Citation (APA):

Chakrabarti, M., Pedersen, H. C., Petersen, P. M., Poulsen, C., Poulsen, P. B., \& Dam-Hansen, C. (2016). Highflux focusable color-tunable and efficient white-light-emitting diode light engine for stage lighting. Optical

Engineering, 55(8), [085101]. https://doi.org/10.1117/1.OE.55.8.085101

\section{General rights}

Copyright and moral rights for the publications made accessible in the public portal are retained by the authors and/or other copyright owners and it is a condition of accessing publications that users recognise and abide by the legal requirements associated with these rights.

- Users may download and print one copy of any publication from the public portal for the purpose of private study or research.

- You may not further distribute the material or use it for any profit-making activity or commercial gain

- You may freely distribute the URL identifying the publication in the public portal 


\title{
Optical Engineering
}

\section{High-flux focusable color-tunable and efficient white-light-emitting diode light engine for stage lighting}

\author{
Maumita Chakrabarti \\ Henrik Chresten Pedersen \\ Paul Michael Petersen \\ Christian Poulsen \\ Peter Behrensdorff Poulsen \\ Carsten Dam-Hansen
}




\title{
High-flux focusable color-tunable and efficient white-light-emitting diode light engine for stage lighting
}

\author{
Maumita Chakrabarti, ${ }^{\mathrm{a}, \boldsymbol{}}$ Henrik Chresten Pedersen, ${ }^{\mathrm{a}}$ Paul Michael Petersen, ${ }^{\mathrm{a}}$ Christian Poulsen, ${ }^{\mathrm{b}}$ \\ Peter Behrensdorff Poulsen, ${ }^{a}$ and Carsten Dam-Hansen ${ }^{a}$ \\ ${ }^{a}$ Technical University of Denmark, Department of Photonics Engineering, Frederiksborgvej 399, 4000 Roskilde, Denmark \\ bBrother, Brother \& Sons ApS, Amager Strandvej 50, 2300 København S, Denmark
}

\begin{abstract}
A color mixing light-emitting diode (LED) light engine that can replace 2-kW halogen-Fresnel spotlight with high-luminous flux in excess of $20,000 \mathrm{~lm}$ is reported for applications in professional stage and studio lighting. The light engine focuses and mixes the light from 210 LEDs of five different colors through a microlens array (MA) at the gate of $\varnothing 50 \mathrm{~mm}$. Hence, it produces homogeneous color-mixed tunable white light from 3000 to $6000 \mathrm{~K}$ that can be adjustable from flood to spot position providing $10 \%$ translational loss, whereas the corresponding loss from the halogen-Fresnel spotlight is 37\%. The design, simulation, and optimization of the light engine is described and compared to the experimental characterization of a prototype. The light engine is optimized through the simulated design of reflector, total internal reflection lens, and MA, as well as the number of LEDs. An optical efficiency of $59 \%$ and a luminous efficacy of $33 \mathrm{~lm} / \mathrm{W}$ are achieved, which is three times higher than the 2-kW halogen-Fresnel spotlight. In addition to having color rendering of color rendering index $R_{a}>85$ and television lighting consistency index $12>70$, the dimmable and tunable white light can be color controlled during the operational time. ๑ 2016 Society of Photo-Optical Instrumentation Engineers (SPIE) [DOI: 10.1117/1.OE .55.8.085101]
\end{abstract}

Keywords: optoelectronics; geometric optical design; illumination design; microstructure fabrication; light-emitting diodes; optical engineering.

Paper 160389 received Mar. 18, 2016; accepted for publication Jul. 8, 2016; published online Aug. 3, 2016.

\section{Introduction}

The traditional light sources such as high-intensity incandescent/tungsten halogen sources ${ }^{1,2}$ have been used earlier on, particularly for studio or stage lighting. The electrical power requirements for such lights are substantially high $(\sim 2 \mathrm{~kW})$ for illuminating the stage sufficiently with more than $20,000 \mathrm{~lm}$ luminous flux $\left(\Phi_{V}\right)$. Thus, those luminaires produce large amounts of heat and infrared radiation during operation and are not energy efficient. Also, such lights require multicolor mechanical/optical filter devices, which is a subtractive method of color filtering to provide effective changes in the projected lighting color that ultimately makes the luminaire even less efficient. In addition, the range of colors in such light sources is limited. In the present scenario, people try to avoid problems in the traditional stage lights by replacing the light source with light-emitting diodes (LEDs) due to the improvement in efficiency and performance reliability. ${ }^{3-5}$ Arrays of multicolor LEDs can be controlled selectively with certain intensity providing a wide range of tunable colors and light intensities. ${ }^{6-8}$ However, there are several LED luminaires available in the market, which use white LEDs with filter as a subtractive color mixing. A more efficient way of doing color mixing is the additive method. ${ }^{9}$ Some LED luminaires use color beams from individual-colored LEDs through lenses and a color mixing mechanism is achieved by overlapping the different color beams into each other. ${ }^{10}$ The mixing of three-colored LEDs (red-green-blue) and four-colored LEDs (red-greenblue-amber or red-green-blue-white) are a few examples

*Address all correspondence to: Maumita Chakrabarti, E-mail: macha@ fotonik. dtu.dk of similar kinds of luminaires using this technique. ${ }^{10,11}$ Although the four-colored LEDs are providing better color rendering index (CRI) than a luminaire based on only three colors, they both have a shortcoming of colored shadows. ${ }^{11}$ There are other possibilities to get unlimited color mixing by use of packages of multicolored LED chips, integrated with a single lens. ${ }^{12}$ However, there is a tradeoff as they cannot produce a high $\Phi_{V}(\sim 20,000 \mathrm{~lm})$ from one single package. Thus, in general, it is required to have a multicolored LED luminaire with a high-lumen output and simultaneously a high CRI. In addition, it is also necessary to have very compact illumination devices to be able to handle and operate them, which is difficult to achieve when more light sources are being integrated into the same illumination device. Due to étendue limitations, ${ }^{13-15}$ the task to combine light from high viewing angle ( $\sim 110$ to $145 \mathrm{deg})$ light sources ${ }^{16}$ into a narrow focusable light beam is complicated. For a large size of collection optics, the LED source can be treated as a point source. However, the point source approximation cannot hold in the case of using compact collection optics. One solution to this problem could be the case where an optical system collects the light from the optical gate and is adapted to image the optical gate at a target surface. ${ }^{10,17}$ Recently, the lighting architecture of such a system is also described in a patent by Jørgensen. ${ }^{18}$ However, these designs cannot sufficiently produce light with more than $10,000 \mathrm{~lm}$.

In this paper, we report a multicolored LED light engine, which is capable of producing focusable and tunable white light of $>20,000 \mathrm{~lm}$ by using a color mixing technique $\mathrm{e}^{10,19,20}$ of beams coming from 210 LEDs using five different LED 
colors. The idea is to replace the 2-kW halogen-Fresnel spot lamp, which requires a total luminous flux of $20,000 \mathrm{~lm}$, with an improved luminous efficiency. The work presented here is a combination of optical simulation and experimental validation of the design by making a prototype of the light engine and further the experimental characterization of the optical components used in the light engine. The design is basically a folded optical system, utilizing a reflector to direct the collimated beams from the large number of LEDs toward the optical gate of the light engine. A double-sided microlens array (MA) is used for efficient color mixing of the beams arriving at different angles to the gate. The design and cost-effective production method for the MA is described by Chakrabarti et al. ${ }^{21}$ The major concern for the optimization of the light engine is the large number of LEDs positioned far from the gate for which the light has a large angle of incidence at the gate. A calculation of étendue for the individual light sources, total internal reflection (TIR) lens, and the light engine are quantified to evaluate the optical efficiency. The optical efficiency, performance, and the size of the light engine are considered in the optimization looking at the main optical components, such as the size and shape of the TIR lenses, gate, and reflector. Two different sizes of TIR lenses for collimation of the LEDs are designed and produced for the prototype and experimental investigation has been done especially by using blue and white LEDs.

By adjusting the light output from each color of LEDs, the light engine can tune the light output from 3000 to $6000 \mathrm{~K}$. The colorimetric stability of the light output from the light engine has been described by Chakrabarti et al. ${ }^{22,23}$ The prototype is analyzed experimentally for evaluation of total flux (spectral and luminous), colorimetric parameters, beam uniformity, and optical loss for each optical component used in the light engine. To compare the functionality of the light engine to the halogen-Fresnel spot lamp, an experimental comparison of the intensity distributions in flood and spot positions are given. Results on the prototype are supplemented with optimized simulated designs of the light engine.

Section 2 will describe the optical luminaire design in detail.

\section{V8 Light Engine}

An optical architecture for a multicolored LED light engine, called "V8 light engine" is reported. It involves design, optimization by computer modeling using Zemax, experimental characterization, and analysis of the output beam from the light engine to get a uniform homogeneous beam spot in color and intensity. The optical loss and the total $\Phi_{V}$ from the light engine are also thoroughly investigated.

\subsection{Optical Design}

The V8 light engine comprises four-colored LEDs (royal blue, blue, green, and red) supplemented by a warm white LED at $3000 \mathrm{~K}$. The schematic diagram of the light engine is seen in Fig. 1(a), which consists of (1) printed circuit board (PCB), (2) LEDs, (3) TIR lenses, (4) parabolic reflector, (5) optical gate, (6) MA, and (7) Fresnel lens. The parabolic reflector and the TIR lenses are used as primary optics. The MA and the Fresnel lens are used as secondary optics. The optical gate is surrounded by different colored LEDs and TIR lenses, which are sitting on the PCB, making a hexagonal pattern of LED cluster of seven rings inside the V8 light engine [as seen in Fig. 1(b)]. The beam from the individual LEDs is quasicollimated by the TIR lens. The quasicollimated beam is reflected subsequently by the parabolic reflector and is directed toward the optical gate, which is situated at the focal plane of the reflector. The optical gate is acting as an aperture. The hexagonal patterned double-sided convex MA acts as a Kohler integrator ${ }^{24}$ to combine a large number

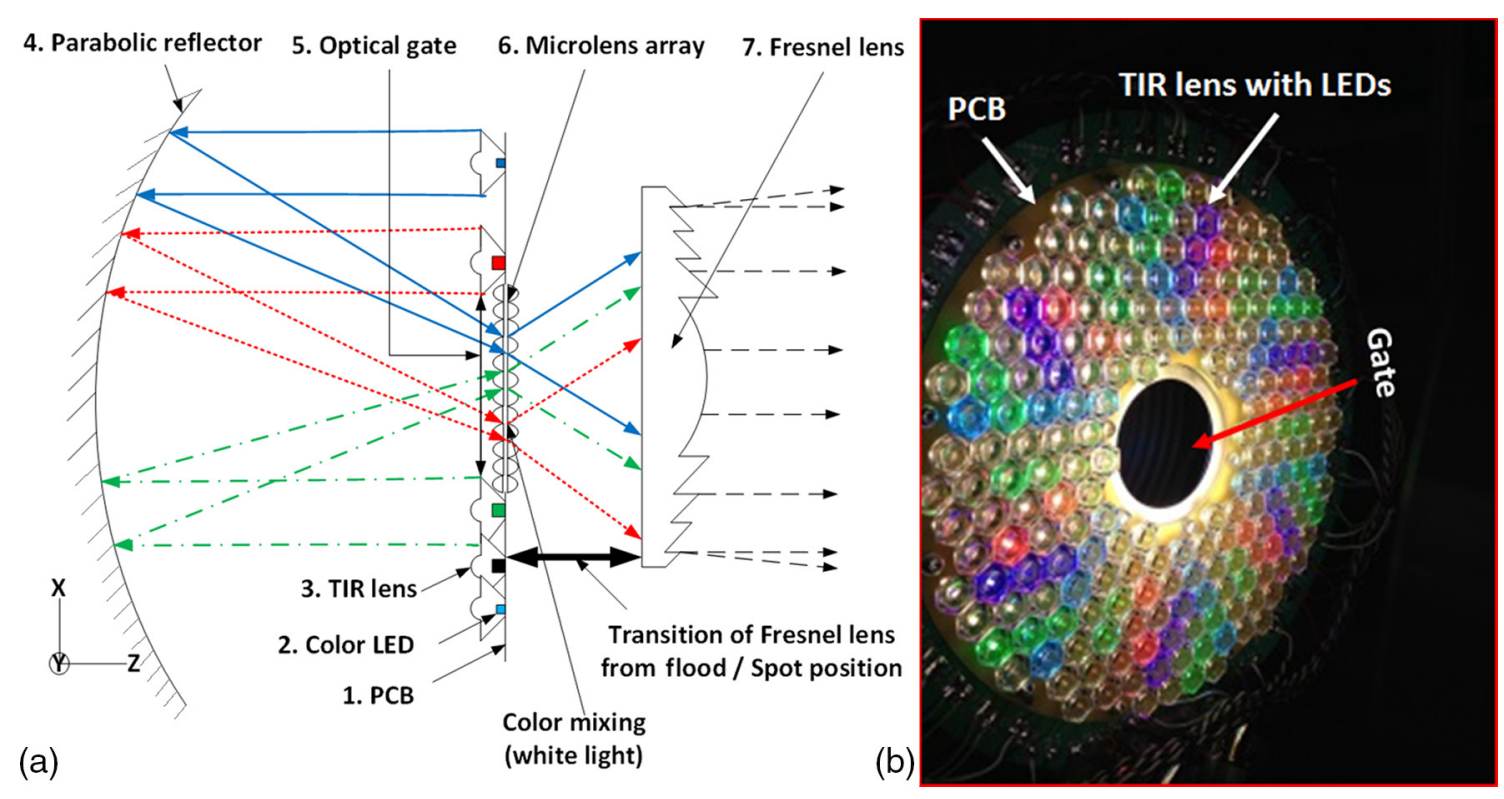

Fig. 1 (a) Schematic diagram of the V8 light engine design (not in scale) where the gate is placed at the focal plane of the reflector; (b) Photo of the PCB of the prototype with 210 operating LEDs with individual TIR lenses, showing the distribution of the four-colored and white LEDs on the PCB forming seven rings (hexagonal pattern); The aperture at the center of the PCB is $\varnothing 50 \mathrm{~mm}$ forming the gate and MA is placed at that position. 
of quasicollimated beams from the individual-colored LEDs arriving at the MA at different angles. The output of the MA is an overlapping of many diverging beams from the individual microlenslets with a divergence angle determined by the numerical aperture (NA) of microlenslets. This constructs a diverging and homogenous color-mixed beam from the MA. The functionality and the experimental performance of the MA, made by polymethyl methacrylate (PMMA), are explained in detail by Chakrabarti et al. ${ }^{21}$ The output from the light engine obstructs by the acceptance angle of the MA. Thus, beams, which are at a higher angle than the acceptance angle of the MA, incident at the gate cannot be transmitted by the MA; thereby increasing the optical loss of the light engine. The acceptance angle can be increased by increasing the NA of the microlenslet. Thus, to obtain a higher acceptance angle and a better color mixing in terms of color homogenization from the light engine, the microlenslet parameters of MA are optimized, which has been reported in the mentioned article. ${ }^{21}$ The working performance of the MA is simulated and experimentally evaluated at the article and the optimized acceptance angle of the MA is $\pm 40 \mathrm{deg}$. The fabrication tool of replicating the MA structure is proposed and demonstrated by making a prototype of MA, which is also reported by Chakrabarti et al. ${ }^{21}$ The fabrication tool as well as the process of making the microlens structure indicates the easy and inexpensive way of producing the MA. The light engine mixes the color from the colored LEDs with warm white LEDs to produce color tunable white light (3000 to $6000 \mathrm{~K}$ ), which can be adjusted in intensity by dimming. Finally, the Fresnel lens is acting as objective lens to collect the color-mixed white light from the MA to provide light output for a spot and a flood position. The translation of the Fresnel lens toward the gate can change the focusable spot position to the flood setting. The light output is stabilized by the control mechanism, which is reported in another article by Chakrabarti et al. ${ }^{22}$

The hexagonal patterned seating arrangements of 210 LEDs on the PCB inside the prototype are divided into six pie segments, where the four-colored and the white LEDs are distributed in a pattern as shown in Fig. 1(b). The individual TIR lens, made by PMMA is mounted on each LED [Fig. 2(a)] by inserting its three legs into holes in the PCB and is fixed by applying glue. The TIR lens optimization was carried out by only focusing on the design of TIR surfaces shown in Fig. $2(\mathrm{~b})^{25}$ to extract the maximum quasicollimated light output from the white LED and fabricated by the injection mold technique. The rays designated by " 1 " and " 2 " are coming from the center point and the edge of the LED, respectively. The intensity pattern of the LED beam at the virtual detector after collimated from the simulated TIR lens [Fig. 2(c)] is seen in Fig. 2(d). Water cooling is used in the prototype of the V8 light engine to dissipate the excess heat generated within the LED cluster. However, the

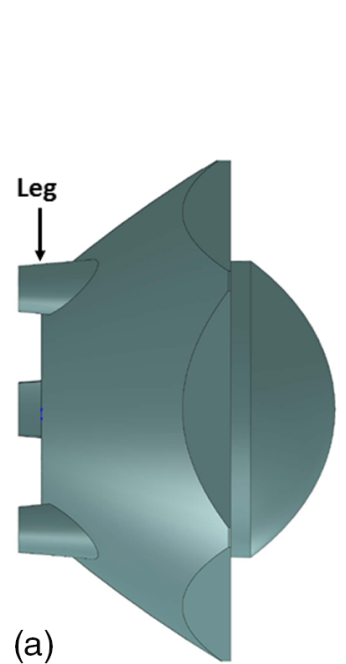

(a)

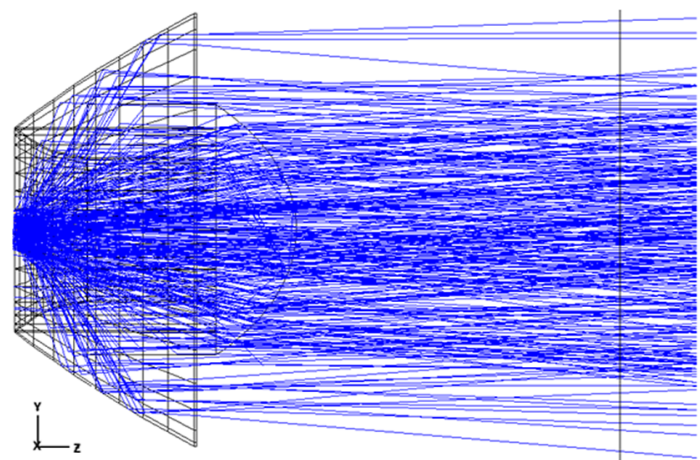

(c)

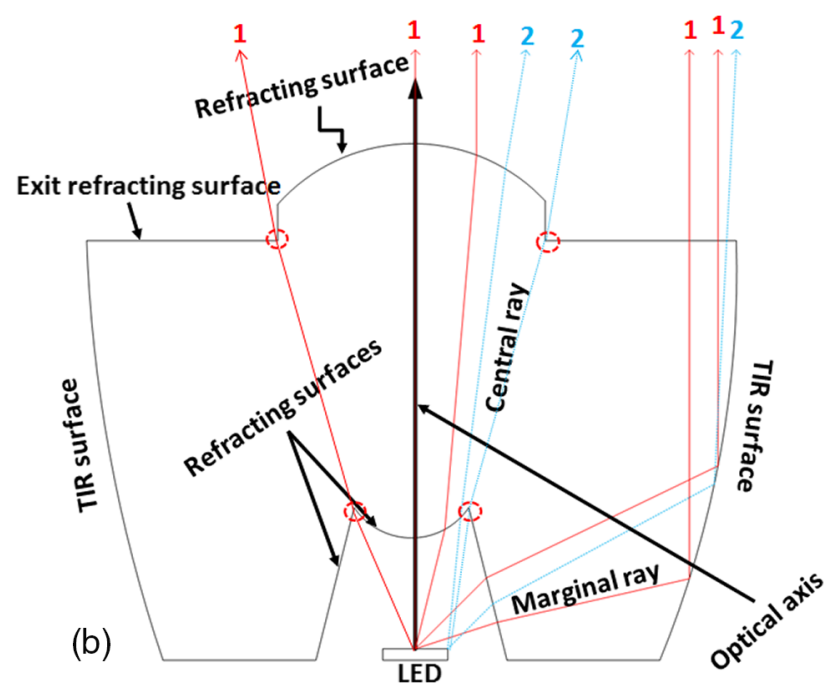

Fig. 2 (a) Side view of TIR lens showing the three legs of $1 \mathrm{~mm}$, (b) indicating TIR surfaces with ray paths (not in scale), (c) ray tracing at simulated TIR lens, and (d) intensity distribution from LED after collimated from simulated TIR lens. 
final product would provide the active cooling by heat pipes and a fan. The digital multiplex signals (DMX) 512A is used to drive the LED channels using a DMX decoder/driver.

\subsection{Optical Model and Simulation}

The purpose of doing optical simulation of the optical design is to provide design flexibility and optimization of light throughput. It also helps to finalize the position and size of the optical components. Optical simulation of the V8 light engine design has been done using by Zemax optical software.

To simulate the V8 light engine design, the parabolic reflector and the TIR lenses, corresponding CAD files are loaded as .stl files. The material of the TIR lens is defined as the PMMA while the reflector is defined as a mirror, where reflection losses are considered by defining coating. The other optical components (e.g., PCB, gate, and MA) are defined as Zemax objects. Initially, the design consisted of seven rings on which 210 LEDs were placed as seen in Fig. 3. The ray files for the LEDs are considered into the Zemax file from the respective source files, which is available from the corresponding supplier's websites. Each colored LED with their respective ring position was simulated separately through the TIR lens to investigate the radiation loss as well as the total light output from an individual scenario of color tuning between 3000 and $6000 \mathrm{~K}$. However, to ease the simulation process for the entire V8 light engine, instead of simulating 210 LEDs, one white LED with an individual TIR lens at each ring is considered. This method saves computational time as well as reduces the complexity. The ray file with 500,000 rays for each white LED is considered into the Zemax file from the respective source file. The total light output was finally estimated by considering the other colored LEDs with their respective position in the ring and their corresponding light output. The total light output from the simulation was investigated by placing a virtual detector as a screen at far-field distance $(\sim 5 \mathrm{~m})$ from the optical gate, where the exit cone was $\pm 40 \mathrm{deg}$. At far-field distance, the light distribution of the resulted beam is unchanged. The parameters of the reflector had been optimized by visual optimizer using Zemax software while the image of the spot for the illumination on the lighting trace detector (placing at the gate) had been optimized according to the highest intensity by the changed parameters (such as focal length, radius of curvature, and diameter) of the parabolic reflector. The TIR lens and the $\mathrm{MA}^{21}$ have been optimized to obtain high light throughput from the optical gate. Section 3 will show the outcome of the optimization.

\subsection{Prototype and Experiment}

To demonstrate the optical design of the V8 light engine, we have developed the prototype of that design. The experimental investigation of the prototype follows the Commission Internationale de lÉclairage (CIE) test standard. ${ }^{26}$ The total spectral flux from the prototype as well as from the individual colored LED was measured by using $2 \pi$ port of an integrating sphere (IS) of $1 \mathrm{~m}$ in diameter equipped with an array spectro-radiometer (CAS-140CT). The light was measured in forward flux condition and a self-absorption correction was applied. The LED system was measured at different settings of correlated color temperature (CCT), such as 3000, 4500, and $5800 \mathrm{~K}$. The LEDs were driven with the required current by DMX control. The temperature of the system stabilized after $29 \mathrm{~min}$ at $42^{\circ} \mathrm{C}$ and measurements were done at stable flux conditions. From these measurements, the total spectral flux $\Phi_{V}$ and colorimetric parameters, e.g., CCT and distance from Planckian locus $\left(D_{u v}\right)$ were calculated. The angular distribution of the luminous intensity of the prototype was measured by two different methods to get measurement consistency. For one measurement process, a horizontal $c$-type manually controlled gonio-spectrometer, equipped with an optical probe with diffuser (EOP-146) was used for the irradiance measurement. The detector was fixed at its position and the prototype of the V8 light engine was rotating with a specific angle. The measurement distances was $8.65 \mathrm{~m}$ and the diameter of the luminous area was $D=180 \mathrm{~mm}$. The measurement distance was $48 \times \mathrm{D}$, and thus, the measurement was done in the far field. ${ }^{26}$ For the $100 \%$ DMX setting, the measurements were done for the flood and spot position of the V8 light engine. For the other measurement process, the photometer (PRC Type 211) is placed at different angular positions with respect to the prototype of the V8 light engine and the
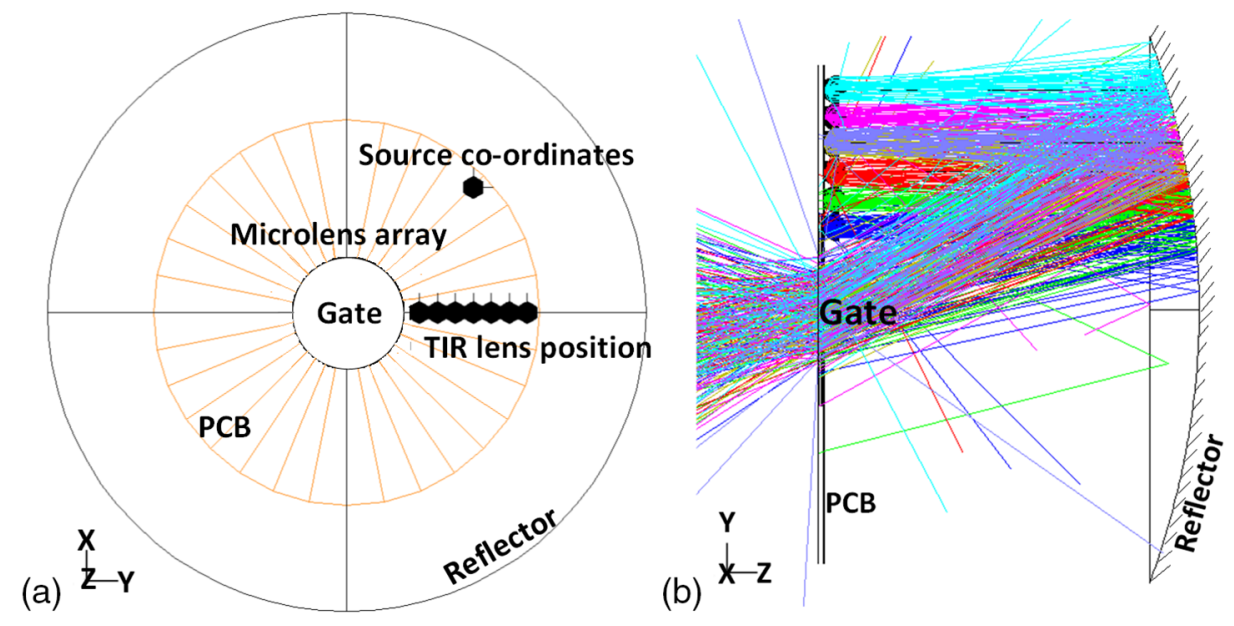

Fig. 3 (a) Top view of TIR lens position on the PCB in simulation software and (b) color mixing of light coming out from the gate. 


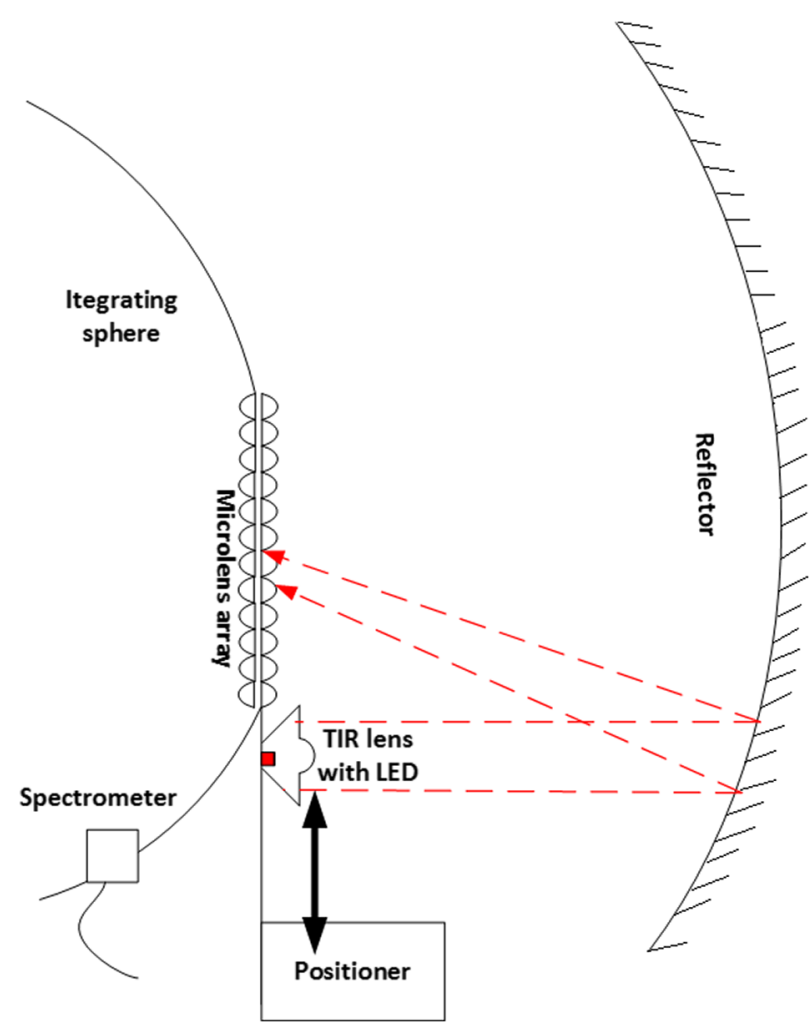

Fig. 4 Loss characterization measurement of LED, TIR lens, and reflector with respect to the position in the light engine. Gate and MA is placed at the port of the IS.

Table 1 Optical loss (\%) for the individual color LEDs at the V8 prototype.

\begin{tabular}{|c|c|c|c|c|}
\hline LEDs & $\begin{array}{l}\text { LED } \\
\text { numbers } \\
\text { used in } \\
\text { prototype }\end{array}$ & $\begin{array}{l}\Phi_{V}(\mathrm{Im}) \text { at } \\
\text { individual } \\
\text { color LEDs + } \\
\text { TIR lens at } \\
100 \% \text { DMX }\end{array}$ & $\begin{array}{l}\Phi_{V}(\mathrm{Im}) \text { at } \\
\text { individual color } \\
\text { LEDs at } 100 \% \\
\text { DMX inside } \\
\text { the prototype }\end{array}$ & $\begin{array}{l}\text { Loss }(\%) \text { for } \\
\text { individual color } \\
\text { LEDs inside } \\
\text { the prototype }\end{array}$ \\
\hline Royal blue & 18 & 466 & 238 & 51 \\
\hline Blue & 24 & 1396 & 688 & 49 \\
\hline Red & 12 & 1224 & 352 & 29 \\
\hline Green & 30 & 5597 & 1675 & 30 \\
\hline White & 126 & 22,960 & 8375 & 36 \\
\hline Total & 210 & 31,643 & 11,327 & 39 \\
\hline
\end{tabular}

corresponding illuminance was measured at that angular position. Afterward, the intensity at different angular positions was calculated from the corresponding illuminance values. To measure the beam homogeneity in CCT and CRI of the prototype of the V8 light engine, a handheld spectrometer (GS-1150) was used at different angular positions. The beam was projected on a screen at far-field distance $>27 \times D$ $(\sim 5 \mathrm{~m})$. The experimental results are plotted and discussed in Sec. 3. We have separately tested the reflector for investigation of the reflector loss by illuminating the reflecting surface by collimated white light. We have also characterized the optical loss for each LED and TIR lens with respect to their position in the ring from the optical gate [as seen in Fig. 3(a)] using the same reflector that has used in the prototype. Figure 4 shows the experimental setup for the same where one TIR lens is mounted on one LED, is positioned on a linear translator helping to place the LED and TIR lens at different positions with respect to the optical gate. Light output from the TIR lens after being reflected by the reflector at each LED position was measured by using $2 \pi$ port of $1-\mathrm{m}$ IS.

\section{Results and Discussion}

This section will provide the dimension of the optical system including the modified values of the corresponding optical components and étendue calculations for the light engine. It also discusses the simulation and the experimental results for the total light output from the optical system after characterizing the optical losses for the components used in the light engine. The total light output and the light intensity distribution (LID) from the light engine are finally compared with the halogen-Fresnel spotlight.

\subsection{Simulation and Experimental Results}

Table 1 compares the forward flux measurement at $100 \%$ DMX from the individual-colored LEDs transmitted from the TIR lens using 2-m IS with the forward flux measurement at $100 \%$ DMX from the individual-colored LEDs from the prototype using 1-m IS. Total loss inside the prototype is $20,316 \mathrm{~lm}$. However, the loss was decreased to $18,354 \mathrm{~lm}$, when the quality of the reflector surface was modified. After using the modified reflector, the optical efficiency of the prototype was $\sim 42 \%$. The position of the LEDs, with respect to the gate, influences the loss in the prototype, which is discussed later.

By using the additive color mixing of four-colored LEDs to supplement and change the SPD from a warm white LED at $3000 \mathrm{~K},{ }^{26}$ tunable CCTs from 3000 to $6000 \mathrm{~K}$ were obtained. Table 2 shows an example of the experimentally produced three settings of CCTs after using different DMX proportion of color LEDs in percentage. It also indicates

Table 2 Light output from V8 light engine at three CCT settings.

\begin{tabular}{|c|c|c|c|c|c|c|c|c|c|c|c|}
\hline \multirow[b]{2}{*}{ Target CCT } & \multicolumn{5}{|c|}{ DMX (\%) of LED color components } & \multicolumn{6}{|c|}{ Produced light quality } \\
\hline & Royal Blue & Blue & Green & Red & White & $\mathrm{CCT}$ & $\mathrm{CRI}\left(R_{a}\right)$ & $\mathrm{CRI}\left(R_{9}\right)$ & $D_{u v}\left(10^{-4}\right)$ & TLCI 12 & $\Phi_{V}(\mathrm{Im})$ \\
\hline 3000 & 1 & 0.5 & 17.3 & 100 & 100 & 3018 & 89.2 & 41.9 & -6.6 & 75 & 7901 \\
\hline 4500 & 14.2 & 45.9 & 77.5 & 79.3 & 100 & 4514 & 91.1 & 89.1 & 0.88 & 91 & 9295 \\
\hline 5800 & 22.6 & 90.3 & 100 & 3.5 & 100 & 5814 & 88.5 & 76.2 & 1.5 & 87 & 9315 \\
\hline
\end{tabular}


the color quality of the light by evaluating the $\mathrm{CRI}^{27}$ and television lighting consistency index (TLCI 12) ${ }^{28}$ scales. According to the application, the score for general CRI $\left(R_{a}\right)$, special CRI $\left(R_{9}\right)$, and TLCI 12 are good for three CCT settings. The TLCI 12 score $>85$ requires no correction for camera in studio. Since all $D_{u v}$ values in Table 2 are $<10^{-3}$, the solutions for CCT $4500 \mathrm{~K}$ is located on the Planckian locus and the solutions for 3000 and $5800 \mathrm{~K}$ are white light with pinkish and greenish tint, respectively. The obtained $\Phi_{V}$ in Table 2 for three CCT settings was low because of the losses encountered inside the system. The following paragraphs would characterize different losses inside the system. To enhance the light output from the system, the optimization of the optical components helped to overcome the system losses to some extent.

The parabolic reflected surface is made by two layers. Initially, it is spin coated by hydrogen silsesquioxane and then physical vapor deposition process of aluminum is applied. First, the process of coating was not good and because of that the reflector had a $30 \%$ of reflectance loss from the nonuniformities in the reflector. Afterward, the reflector was modified with a controlled coating of aluminum and the process helped to get a good surface uniformity with reflectance of $\sim 89 \%$, which eventually improved a $24 \%$ on average in $\Phi_{V}$ output at the previously mentioned CCT settings. Therefore, the $\Phi_{V}$ increased from 9315 to $12,420 \mathrm{~lm}$ at $5800 \mathrm{~K}$. Table 3 provides the experimentally measured individual optical loss in \% from the optical components used in the prototype. Thus, for the present scenario, the optical efficiency of the V8 light engine cannot exceed of $\sim 61 \%$. However, by applying the antireflecting coating on the optical components may reduce the inherent optical loss inside the light engine. The prototype of the V8 light engine cannot produce sufficient light output $(>20,000 \mathrm{~lm})$. Therefore, to enhance the optical efficiency of the prototype from $42 \%$, further the design modifications of the optical components are required.

However, before the design modification, it is essential to check whether the light engine does not violate the étendue principle. Table 4 records the individual étendue calculations for light source as explained in Gadegaard et al. ${ }^{10}$ article for

Table 3 Optical loss budget for the used components inside the prototype.

\begin{tabular}{lc} 
Optical component & Optical loss (\%) \\
\hline TIR lens & 12 \\
Parabolic reflector & 11 \\
MA & 8 \\
Fresnel lens & 8 \\
\hline
\end{tabular}

used LED, TIR lens as well as for the optical gate of the prototype. The chip size of the white, the green, and the red LED is the same $(1 \mathrm{~mm} \times 1 \mathrm{~mm})$ whereas the blue and the royal-blue LED have the small size $(0.55 \mathrm{~mm} \times 0.53 \mathrm{~mm})$. So, we have calculated the étendue separately for the white $\left(\mathrm{E}_{\mathrm{whiteLED}}\right)$ and the blue ( $\left.\mathrm{E}_{\text {blueLED }}\right)$ LED, respectively. Similarly, we have calculated étendue for TIR lens as $\mathrm{E}_{\text {whiteTIR }}$ and $\mathrm{E}_{\text {blueTIR }}$, which used the white and the blue $\mathrm{LED}$, respectively. We have also calculated the total étendue for 210 LEDs $\left(\mathrm{E}_{\text {source }}\right)$ and the étendue for the collecting optics $\left(\mathrm{E}_{\mathrm{gate}}\right)$. For each case, the étendue ratio for the collection optics and the light source is above 1 and thus the light engine is not étendue limited. Therefore, there is a freedom for the optimization of the system for the better performance.

We have optimized the reflector size and shape by simulation to obtain more light output from the light engine. It is observed that the parabolic reflector provides higher optical efficiency than a reflector designed by the freeform reflector. This is because the parabolic reflector in overall delivers higher optical efficiencies for the TIR lenses that are near from the optical gate (Fig. 5). However, the goal of the designed free-formed reflector was to provide the highest optical efficiency at the farthest ring position. In Fig. 5, down $X$-axis represents the TIR and the LEDs positions at the rings. The corresponding angles generated at the gate due to the positions of the TIR lenses and the LEDs with respect to the gate are shown at the top $X$-axis in Fig. 5 . However, the optical efficiency decreases gradually for the parabolic reflector when the position of the TIR lens is

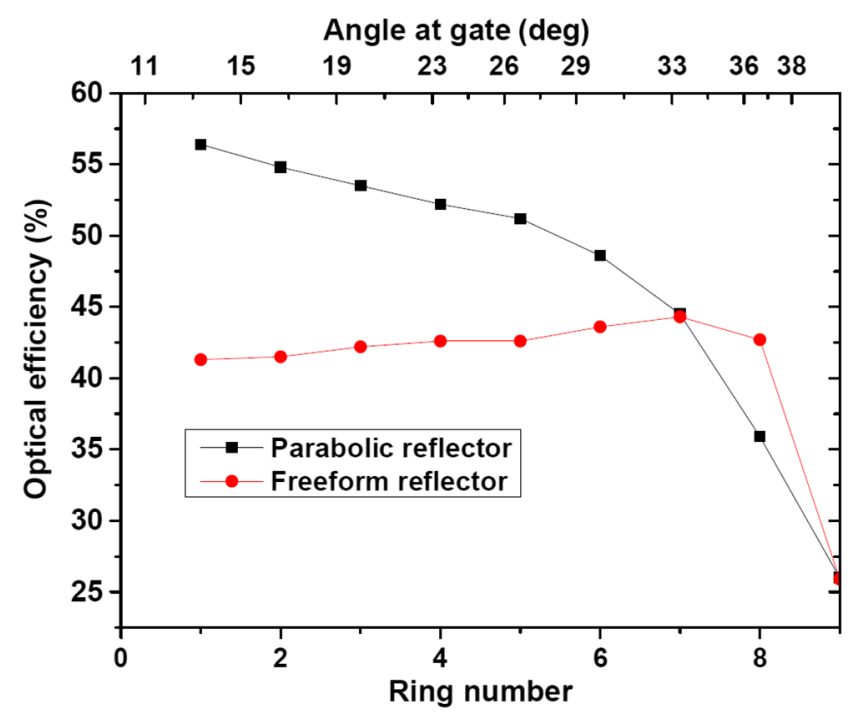

Fig. 5 Simulated optical efficiency of the LEDs positions corresponding to the ring number in the light engine for the parabolic and the freeform reflector. Corresponding angle at the gate is given at the top $X$-axis.

Table 4 Étendue calculations for used LEDs, TIR lens, and optical gates.

\begin{tabular}{|c|c|c|c|c|c|c|c|c|}
\hline $\begin{array}{l}E_{\text {whiteLED }} \\
\left(\mathrm{mm}^{2}\right)\end{array}$ & $\begin{array}{c}\mathrm{E}_{\text {white TIR }} \\
\left(\mathrm{mm}^{2}\right)\end{array}$ & $\begin{array}{l}\mathrm{E}_{\text {white TIR }} / \\
\mathrm{E}_{\text {whiteLED }}\end{array}$ & $\begin{array}{l}\text { EblueLED } \\
\left(\mathrm{mm}^{2}\right)\end{array}$ & $\begin{array}{c}\text { Eblue TIR }_{\left(\mathrm{mm}^{2}\right)} \\
(\mathrm{m}\end{array}$ & $\begin{array}{l}E_{\text {blueTIR }} / \\
E_{\text {blueLED }}\end{array}$ & $\begin{array}{l}E_{\text {source }} \text { for } 210 \\
\text { LEDs }\left(\mathrm{mm}^{2}\right)\end{array}$ & $\begin{array}{l}E_{\text {gate }} \\
\left(\mathrm{mm}^{2}\right)\end{array}$ & $\begin{array}{l}E_{\text {gate }} / \\
E_{\text {source }}\end{array}$ \\
\hline 3.1 & 4.95 & 1.58 & 0.9 & 7.74 & 8.46 & 566 & 3284 & 5.8 \\
\hline
\end{tabular}


far from the gate. Irrespective of position of the TIR lens with respect to the gate, the efficiency is constant until the sevenring position for the free-formed reflector. As seen in Fig. 5, at the eight ring position ( $\sim 97 \mathrm{~mm}$ from the gate), the efficiency is higher for the free-formed reflector compared to the parabolic reflector.

Although the purpose of using the MA in the design is to mix the color uniformly, the incident beam higher than the acceptance angle of the MA cannot come out from the gate window and, therefore, decreases the overall optical efficiency of the system as explained in the article of Chakrabarti et al. ${ }^{21}$ Thus, the higher angle beam $(> \pm 35 \mathrm{deg})$ reflected from the parabolic reflector reaching at the gate, is not allowed to transmit through the gate. To overcome this shortcoming, we have increased the acceptance angle of the MA from \pm 35 to \pm 40 deg by optimizing the width of the single lens, which ultimately increases the NA of the individual lenslet of MA. ${ }^{21}$ This optimization helped to enhance the final light output by $52.5 \%$ compared to the prototype.

The working performance of the two different sizes of TIR lens has been investigated. The experimental setup described in Figs. 4, 6(a), and 6(b) graphically represents the experimentally evaluated optical efficiencies for the white and the blue LEDs using two sizes of TIR lenses. In Figs. 6(a) and 6(b), the down $X$-axis represents the position of the TIR lens and the LED at the ring, whereas the top $X$-axis represents the corresponding angle created at the gate. As seen in the both figures [Figs. 6(a) and 6(b)], the optical efficiency is higher for the both LEDs (white and blue) when the system is assembled without MA. Because the beams that are larger than the acceptance angle (or cutoff angle) of the MA are obstructed by the MA to transmit through the gate. To receive collimated light from the LEDs, we have used TIR lenses. However, there is a divergence of maximum $\sim \pm 5 \mathrm{deg}$ for the white, the red, and the green LEDs and $\sim \pm 7 \mathrm{deg}$ for the blue and the royal-blue LED with respect to the parallel beam, respectively. Due to this divergence, the incoming beam toward the gate after reflection from the parabolic mirror becomes more tilted if the LEDs are situated far from the gate. Also, the viewing angle of the blue LED is higher than the white LED. Thus, the collecting area for the blue LED needs to be larger than the white LED to conserve the étendue and also seen in Table 4. Therefore, the cutoff angle of MA influences more losses for the blue LED than the white LED and that explains why the optical efficiency of the blue LEDs is always higher than the white LEDs while the system is not using an MA.

Further, angular dispersion of the light engine is investigated thoroughly by simulation. It is assumed that a point source is placed at the center of the LED chip. The LED chip is also situated at the center of the TIR lens [Fig. 2(b)]. A lateral movement of the point source by $0.5 \mathrm{~mm}(\Delta x)$ from the center position will lead to a lateral movement, $(\Delta y)$ of the rays at the incident position on the gate where the MA is situated. Therefore, the dispersion $(D)$ of the ray for different ray angles at the gate is evaluated according to
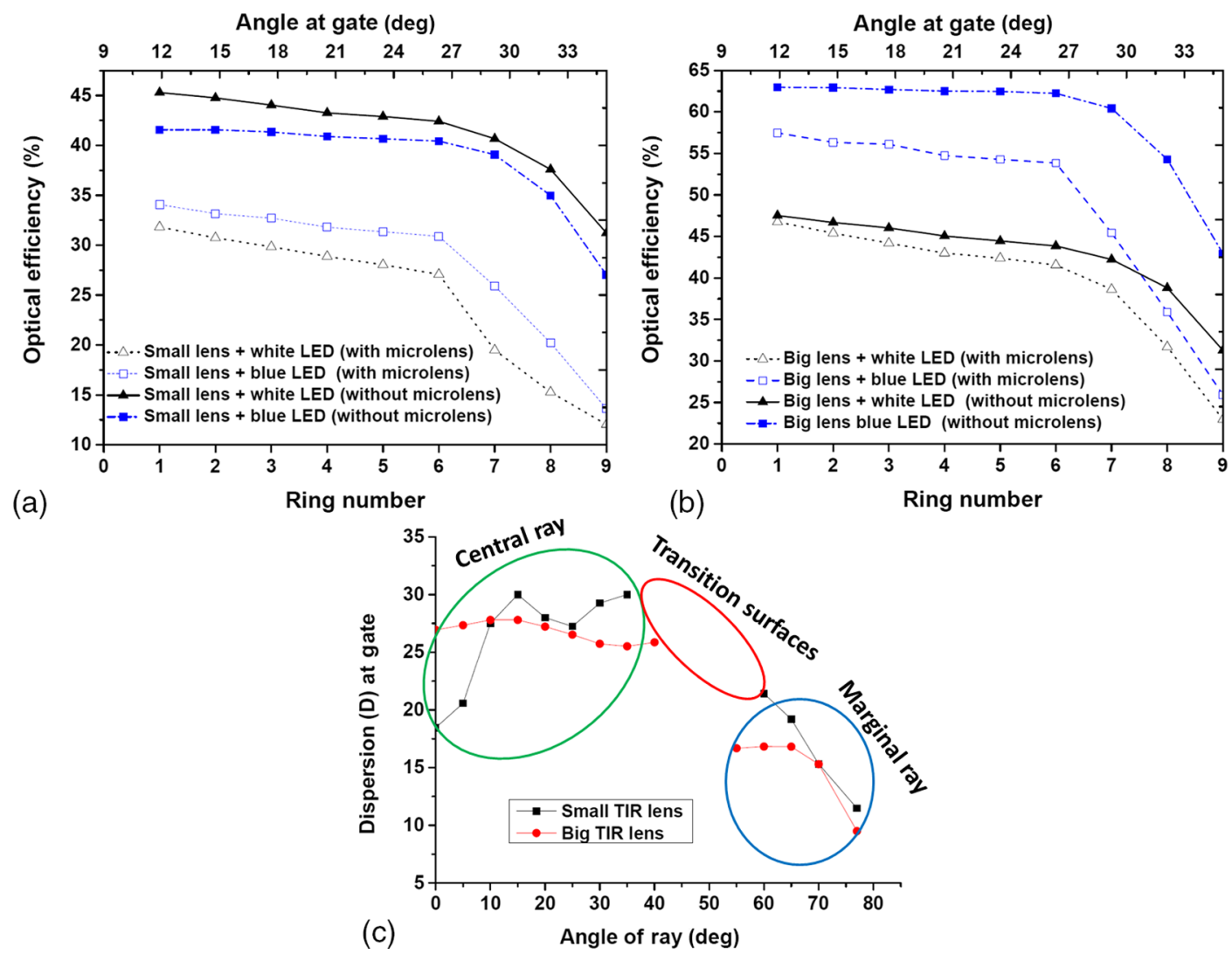

Fig. 6 (a) and (b) Optical efficiency versus LED position given by the ring numbers with respect to the gate for small and big TIR lens, respectively. Corresponding angle at the gate is given at the top $X$-axis. (c) Dispersion sensitivity of ray angles at the gate of the light engine when point source is considered for the simulation. 
$D=\frac{\Delta y}{\Delta x}$

Figure 6(c) graphically represents the dispersion of the different rays for different emission ray angles of the point source. The dispersion of marginal rays (rays with large emission angles hitting the TIR part of the lens) at the gate is smaller than for the central or principle rays [rays with small emission angles, hitting the central part of the TIR lens, see Fig. 2(b)]. Transition rays are those rays that accidentally hit a mixture of the central and marginal areas of the TIR lens and, therefore, are lost [dashed red circles in Fig. 2(b)]. The figure also shows that the bigger size of TIR lens $(12.9 \mathrm{~mm})$ has less dispersion than the smaller size of TIR lens $(11.9 \mathrm{~mm})$. The increased TIR lens size from 11.9 to $12.9 \mathrm{~mm}$ aided to improve the final light output by $49.8 \%$ compared to the prototype. Generally, TIR-based lens collimates the broad beam from the LED down to a more usable narrow beam angle. Now, the output end of the TIR lens has to be large enough that étendue can be conserved, as a narrower angle beam means TIR lens must have a larger emitting area. This explains that the bigger TIR lenses inside the light engine deliver better collimation due to the étendue principle and the better collimation leads to the better performance from the parabolic reflector. Thus, the optimal lens size $12.7 \mathrm{~mm}( \pm 0.2 \mathrm{~mm})$ is used in the modified design. We have optimized the TIR lens size with respect to the illumination from the white LEDs. Thus, there is maximum $\sim 56 \%$ optical efficiency hike for white LED by using bigger size of TIR lenses. Bigger TIR lenses also help the blue LEDs to increase its optical efficiency by $\sim 56 \%$. However, the light extraction from the blue LEDs still needs to be optimized for higher efficiency, which is not done. This is because, by using the same size of TIR lens for all LEDs in the V8 light engine leads to provide a cost-effective solution as well as the simplicity of the design.

The dimensions of the individual optical components used in the prototype and the optimized designs of the light engine ("design 1" and "design 2") are listed in Table 5. The total LED numbers used in the prototype and in the optimized designs are also listed in the same table. There was an investigation on the different gate sizes and finally the gate size of $\varnothing 50 \mathrm{~mm}$ is used. A bigger gate size than $\varnothing 50 \mathrm{~mm}$ cannot effectively enhance the overall light output from the light engine because the bigger gate size leads to increase the loss from the farthest TIR lenses, which are near to the perimeter of the PCB (Fig. 3). To compensate the loss from TIR lenses, the overall optical system size needs to increase, which is not desirable. For example, the gate

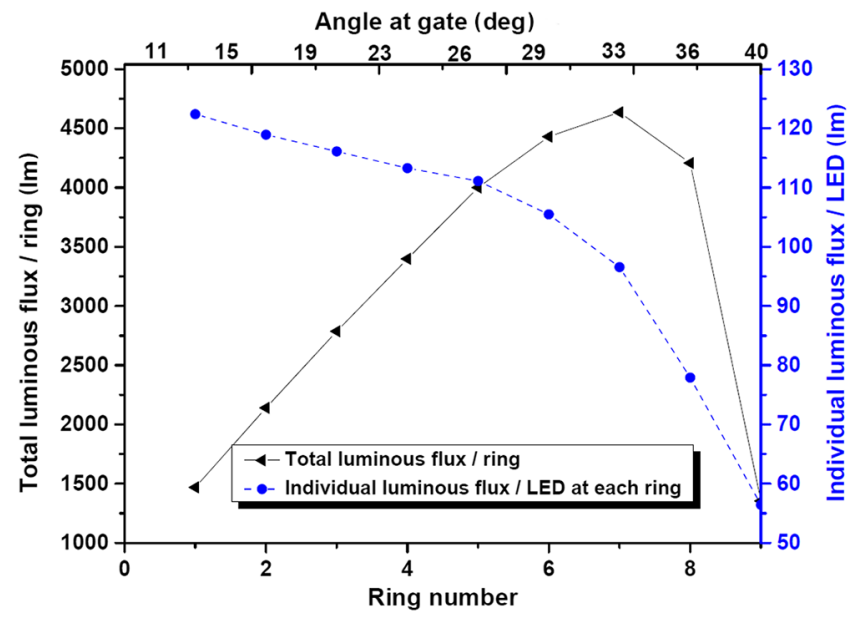

Fig. 7 Total and individual luminous flux pr. ring and LED at each ring position, respectively. Corresponding angle at the gate is given at the top $X$-axis.

size of $\varnothing 55 \mathrm{~mm}$ increases $500 \mathrm{~lm}$ light output than the gate size of $\varnothing 50 \mathrm{~mm}$. However, the reflector size needs to increase by 0.8 times than the previous one. To collect the entire light from the gate, the used Fresnel lens was of $\varnothing 180 \mathrm{~mm}$ with $80 \mathrm{~mm}$ of focal length.

Although we have optimized the MA and the size of TIR lens to enhance the light output from the system, the position of the LED with respect to the gate still influences the LED efficiency. This is because, the beam, which is higher than $\pm 40 \mathrm{deg}$, cannot pass through the gate and increases the loss of the light engine. Thus, the LED efficiency decreases while going away from the gate, which is also indicated in Figs. 6(a) and 6(b). We have simulated the luminous efficacy of the white LEDs at each ring position. The black line with solid triangle in Fig. 7 directs the simulated total $\Phi_{V}$ at each ring after adding the total light output from the white LEDs at each ring position. The individual luminous flux of the white LED, represented by the blue-dashed lines (solid dot in Fig. 7), decreases gradually as the ring number increases (far from gate). However, the total luminous flux increases initially with increasing ring number. This is because the increased ring number gradually gives room to hold more LEDs than the previous one. After the ring number 7, the total luminous flux again decreases as the loss from the LED cannot compensate the effect of increasing number of LED.

The simulated result shows that $\Phi_{V}$ values can be increased after optimization of MA and TIR lens and are able to produce $22,284 \mathrm{~lm}$ from the V8 light engine, which is

Table 5 The dimensions of the optical components used in the V8 lighting design.

\begin{tabular}{|c|c|c|c|c|c|c|c|c|c|}
\hline & \multirow[b]{2}{*}{$\begin{array}{l}\text { Total LED } \\
\text { numbers }\end{array}$} & \multicolumn{3}{|c|}{ Parabolic reflector } & \multirow[b]{2}{*}{$\begin{array}{l}\text { PCB size } \\
\text { (mm) }\end{array}$} & \multirow[b]{2}{*}{$\begin{array}{l}\text { Lens size } \\
\quad(\mathrm{mm})\end{array}$} & \multirow[b]{2}{*}{$\begin{array}{l}\text { Gate size } \\
\quad(\mathrm{mm})\end{array}$} & \multirow[b]{2}{*}{ Ring } & \multirow{2}{*}{$\begin{array}{l}\text { Width of } \\
\text { lenslet for } \\
\text { MA (mm) }\end{array}$} \\
\hline & & $\begin{array}{l}\text { Diameter } \\
(\mathrm{mm})\end{array}$ & $\begin{array}{l}\text { Curvature } \\
\text { (mm) }\end{array}$ & $\begin{array}{l}\text { Focal length } \\
(\mathrm{mm})\end{array}$ & & & & & \\
\hline V8 prototype light engine & 210 & 240 & -380 & 170 & 206 & $11.7 \pm 0.2$ & 50 & 7 & 1.2 \\
\hline Optimized design 1 & 210 & 240 & -330 & 165 & 206 & $12.7 \pm 0.2$ & 50 & 7 & 1.45 \\
\hline Optimized design 2 & 288 & 340 & -400 & 180 & 270 & $12.7 \pm 0.2$ & 50 & 9 & 1.45 \\
\hline
\end{tabular}


having total optical efficiency of 59\%. This optical efficiency can further increase with the $95 \%$ reflectance of the parabolic reflector. ${ }^{29}$ Table 6 shows the improvement of the $\Phi_{V}$ after each step of optimization of optical component and the total $\Phi_{V}$ from the V8 light engine. The increase in number of LEDs from 210 (seven rings) to 288 (nine rings) also increases the final light output from 22,284 to $25,990 \mathrm{~lm}$. However, the total optical efficiency decreases from $59 \%$ to $48 \%$ (as seen in Fig. 7), leading to the increase of overall size and weight accordingly by $25 \%$ and $27 \%$, respectively. In addition to this, the difficulty of mechanical design also increases since making such a large reflector is complicated and costly.

Due to time restrictions as well as the budgetary limitation, the second prototype was not made after the design optimization. Figure 8 shows the experimental validation using experimental setup explained in Fig. 4 with respect to the corresponding simulation, where the optical efficiency of the white LED transmitted through the big TIR lens is characterized inside the optical system at different ring position. The average deviation in optical efficiency of the light engine between the simulation and the experimental results is

Table 6 Improvement in total $\Phi_{V}$ and luminous efficacy in flood position compared to the prototype after optimization in simulation in three steps.

\begin{tabular}{lcccc} 
& & $\begin{array}{c}\text { After MA } \\
\text { Parameters }\end{array}$ & $\begin{array}{c}\text { After TIR } \\
\text { lens size }\end{array}$ & $\begin{array}{c}\text { Increased } \\
\text { number of } \\
\text { LEDs from }\end{array}$ \\
\hline Total $\Phi_{V}(\mathrm{Im})$ & 11,327 & 16,624 & 22,284 & 25,990 \\
optimization & & 5297 & 5660 & 3706 \\
$\begin{array}{l}\text { Improvement } \\
(\mathrm{Im})\end{array}$ & - & & & \\
$\begin{array}{l}\text { Luminous } \\
\text { efficacy }(\mathrm{Im} / \mathrm{W})\end{array}$ & 17 & 25 & 33 & 29 \\
\hline
\end{tabular}

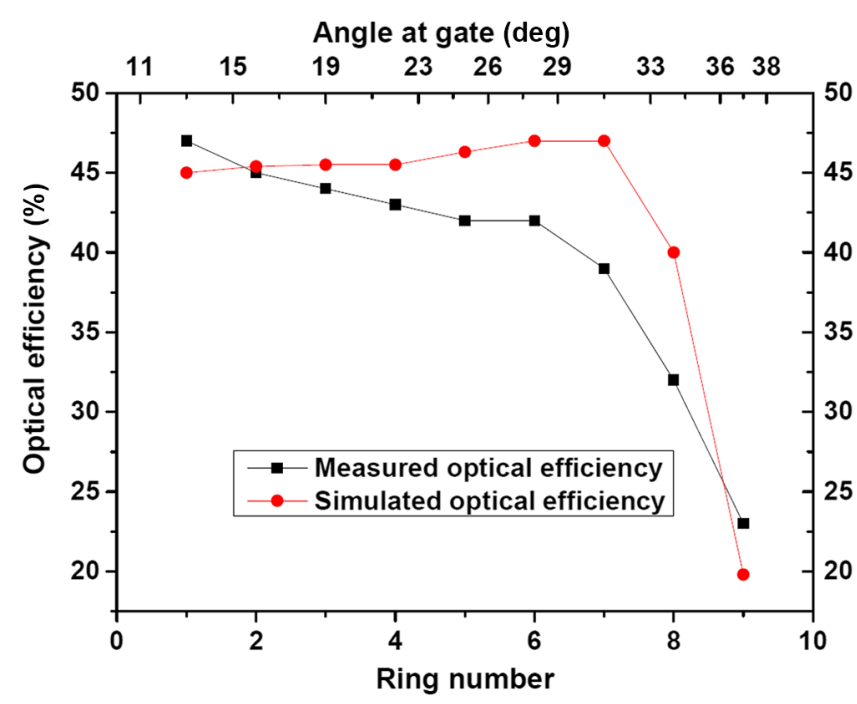

Fig. 8 The comparison between simulation and experimental results considering the optical efficiency of white LED through the TIR lens at each ring position. Corresponding angle at the gate is given at the top $X$-axis. $\sim 9.7 \%$ whereas our measurement uncertainty for the experiment is $\sim 6 \%$.

\subsection{Beam Homogeneity}

Figure 9(a) represents the experimental results for the light intensity at flood position from the prototype measured by the photometer (PRC Type 211) and the manually controlled gonio-spectrometer, respectively (described in Sec. 2.3). Both the measurements show that the full width half maximum (FWHM) of the beam angle is $\sim 60 \mathrm{deg}$ and the uniform intensity is found within $\pm 20 \mathrm{deg}$ [Fig. 9(a)] with a variation of $\sim 11 \%$. There is $25 \%$ variation in intensity within the entire beam size (FWHM) in the measurement by photometer. The data points for the measurement by gonio-spectrometer were less and thus the intensity variation was not observed. The handheld spectrometer (described in Sec. 2.3) is used to investigate the beam homogeneity in CCT and CRI [Fig. 9(b)], and also in $u^{\prime}, v^{\prime}$ (CIE 1976 chromaticity coordinates) parameters [Fig. 9(c)]. The measurement shows the uniformity in CCT, CRI, $u^{\prime}$, and $v^{\prime}$ are within $\pm 23 \mathrm{deg}$, where the variation is $\Delta u^{\prime} v^{\prime}=0.0010$. Due to the measurement uncertainty of low light level, the both end of the graphs in Figs. 9(b) and 9(c) show the large variation of $\Delta u^{\prime} v^{\prime}=0.0047$.

\subsection{Comparison Between V8 Light Engine and Traditional Lighting}

We have measured the light output from the halogen-Fresnel spotlight $(2 \mathrm{~kW})$ to compare with the light output from the prototype of V8 light engine $(0.6 \mathrm{~kW})$. Figure 11 shows the comparison of the normalized LID between the halogenFresnel spotlight and the prototype of the V8 light engine. As seen in Fig. 10(a), the distributions of the halogenFresnel lamp and the prototype of the V8 light engine are similar for the flood position. Due to the MA at the gate in the V8 light engine, the distribution is smoother for the prototype of the V8 light engine than the halogen-Fresnel spotlight. However, the gate diameter $(\varnothing 50 \mathrm{~mm})$ limits the V8 light engine to have 1.6 times broader beam at the spot position than the halogen-Fresnel spotlight, which has small size of filament $(\sim 19 \mathrm{~mm})$, shown in Fig. 10(b).

Table 7 shows the comparison between the measured values of the halogen-Fresnel spotlight and the prototype of the V8 light engine. Although the halogen-Fresnel spotlight is producing $\sim 34 \%$ more luminous flux compared to the present prototype of the V8 light engine, it consumes $70 \%$ more electrical energy. The beam angles (FWHM and field angle) of the both lighting systems are also calculated and are shown in the same table. The calculated results show that the V8 light engine is having wider beam than the halogen-Fresnel spotlight as $\varnothing 50 \mathrm{~mm}$ gate size creates the beam angle (FWHM) to be broader $\sim 55$ and $\sim 16 \mathrm{deg}$ at the flood and the spot position, respectively.

We have assumed that the LIDs are same for the both prototype and the simulation of the V8 light engine. Therefore, we have estimated the intensities of the optimized simulated V8 light engine for the flood and the spot positions, which are shown in Table 7. The V8 light engine is having less intensity than the halogen-Fresnel spotlight because of having a wider beam. However, the optimized simulated V8 light engine after the design modification is providing the comparable luminous flux of halogen-Fresnel 

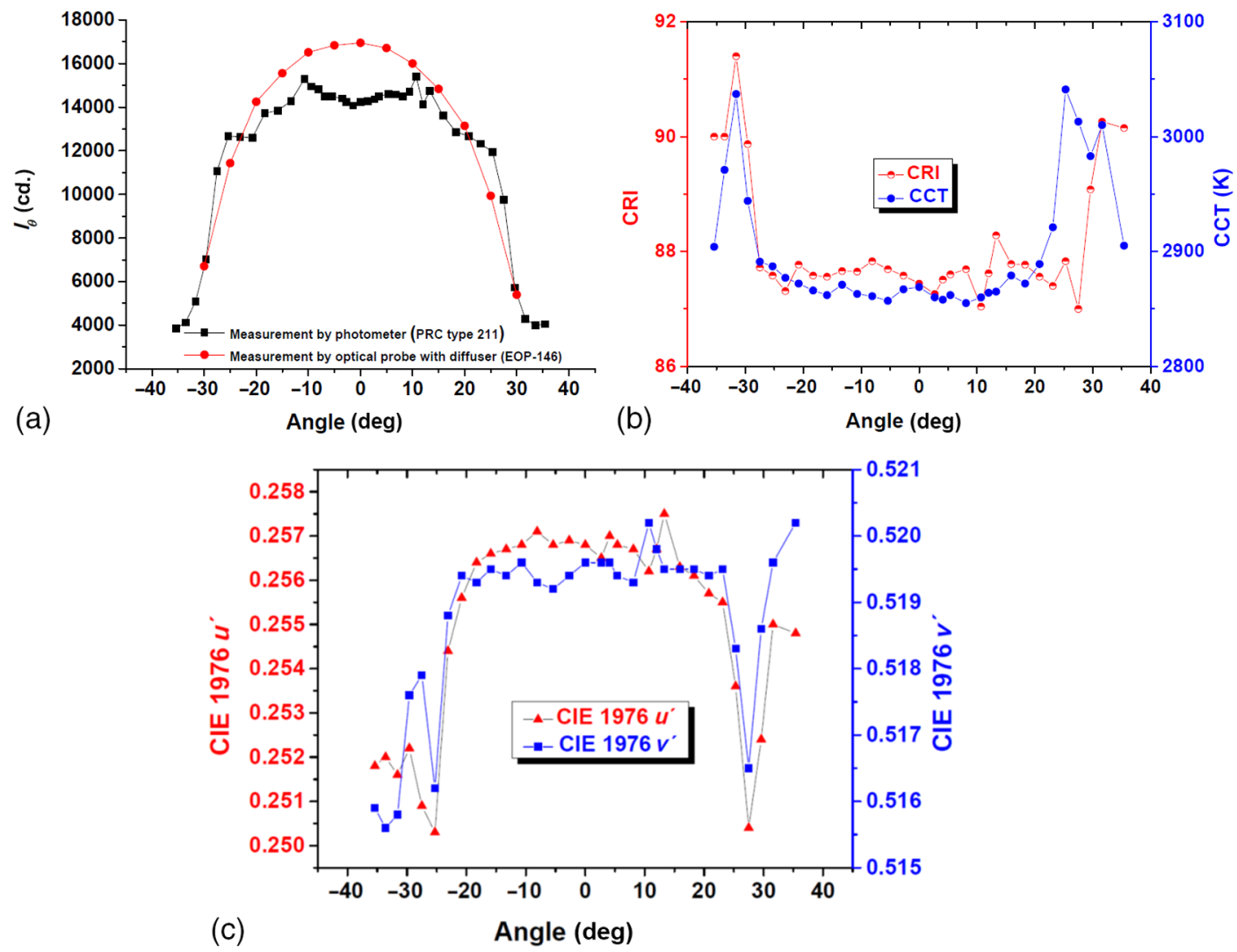

Fig. 9 Distribution of (a) light intensity for two measurements, (b) CRI and CCT, and (c) CIE $1976\left(u^{\prime}, v^{\prime}\right)$ with respect to beam angle.
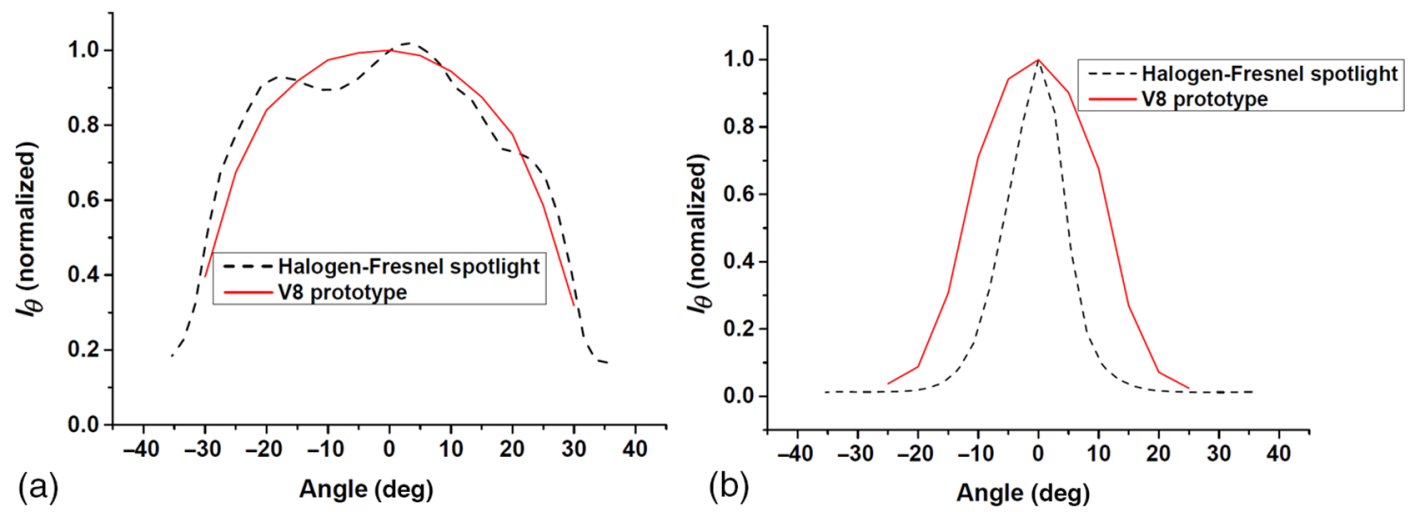

Fig. 10 LID comparison between the halogen-Fresnel spotlight and the prototype of V8 light engine for (a) the flood and (b) the spot position, respectively.

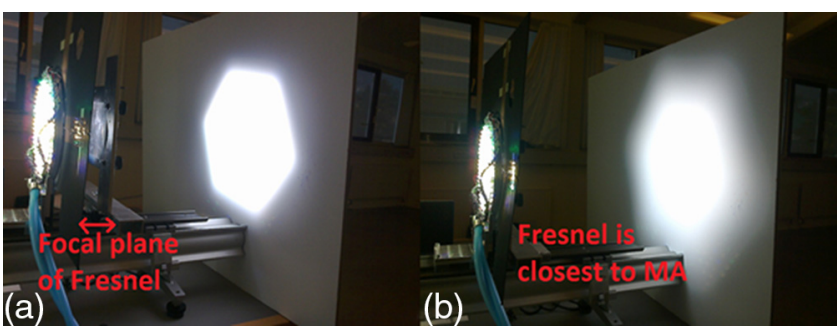

Fig. 11 Photographs of the experimental setup at (a) the spot; (b) the flood light adjustment of the $\mathrm{V} 8$ light engine. spotlight with low electrical consumption. The comparison table also indicates that the loss of the optical energy due to the change from the flood to the spot position is $10 \%$ for the V8 light engine, whereas the losses from the halogen-Fresnel spot lamp is $37 \%$. This reflector in the halogen-Fresnel spotlight cannot collect all the light during the translation from the flood to the spot. However, for the V8 light engine, the size of the Fresnel lens optimized to couple the almost all light during the same translation as mentioned above.

Figure 11 shows the experimental setup for projecting the light at a white cardboard for two configurations (spot and 
Table 7 Measured total $\Phi_{V}$, luminous efficacy, intensity and beam angle for the 2-kW halogen-Fresnel spotlight, the V8 prototype light engine, and corresponding values for the optimized simulated V8 light engine.

\begin{tabular}{|c|c|c|c|c|c|c|}
\hline $\begin{array}{l}\text { Lighting } \\
\text { system }\end{array}$ & $\begin{array}{l}\text { Fresnel lens } \\
\text { adjustment }\end{array}$ & $\Phi_{V}(\mathrm{Im})$ & $\begin{array}{l}\text { Luminous } \\
\text { efficacy } \\
(\mathrm{Im} / \mathrm{W})\end{array}$ & $\begin{array}{l}\text { Intensity } \\
\text { (cd) }\end{array}$ & $\begin{array}{c}\text { FWHM } \\
\text { at } 50 \% \\
(\mathrm{deg})\end{array}$ & $\begin{array}{l}\text { Field } \\
\text { angle } \\
\text { (deg) }\end{array}$ \\
\hline $\begin{array}{l}\text { Halogen- } \\
\text { Fresnel } \\
\text { spotlight }\end{array}$ & Flood & 18,745 & 10 & 33,902 & 50.6 & 69 \\
\hline $\begin{array}{l}\text { V8 } \\
\text { prototype }\end{array}$ & Flood & 12,350 & 19 & 15,680 & 55 & 70.8 \\
\hline $\begin{array}{l}\text { V8 } \\
\text { simulated }\end{array}$ & Flood & 22,284 & 33 & 28,292 & - & - \\
\hline $\begin{array}{l}\text { Halogen- } \\
\text { Fresnel } \\
\text { spotlight }\end{array}$ & Spot & 11,777 & 7 & 237,273 & 10.8 & 24 \\
\hline $\begin{array}{l}\text { V8 } \\
\text { prototype }\end{array}$ & Spot & 11,100 & 17 & 115,494 & 16.2 & 29.2 \\
\hline $\begin{array}{l}\text { V8 } \\
\text { simulated }\end{array}$ & Spot & 20,060 & 30 & 208,721 & - & - \\
\hline
\end{tabular}

flood position) to check the visual appearance. The spot position for the V8 light engine [Fig. 11(a)] is found by placing the gate at the focal plane of the Fresnel lens. To obtain the flood position [Fig. 11(b)], the Fresnel lens is positioned as close as possible to the gate. For the both configurations, the beam looks uniform by visual inspection. However, the shape of the beam is hexagonal due to the hexagonal structure of the MA.

\section{Conclusions}

We have reported a focusable, color-mixed, and tunable white LED stage lighting system, which is capable of producing $\sim 20,000 \mathrm{~lm}$ luminous flux $\left(\Phi_{V}\right)$ by simulation with optical efficiency of $\sim 59 \%$. A double-sided hexagonal patterned MA was used at a gate of $\varnothing 50 \mathrm{~mm}$ for beam homogenization. The design was demonstrated by producing a prototype. The prototype was characterized and investigated experimentally. The optical efficiency obtained from the experimental results validates the simulation with the deviation of $\sim 9.7 \%$. The optical loss from the used optical components is thoroughly investigated. We have also reported a quantitative estimation of étendue of the light engine. The light output from the prototype does not fulfill the high $\Phi_{V}$ requirements $(20,000 \mathrm{~lm})$ from the light engine. Thus, the optical components used in the light engine, such as the reflector, TIR lens, MA, and the number of LEDs are optimized through simulation to achieve the $\Phi_{V}$ requirements of the light engine and enhance the luminous efficacy to $\sim 33 \mathrm{~lm} / \mathrm{W}$, which is three times higher than the $2-\mathrm{kW}$ halogen-Fresnel spotlight. The comparison results between the traditional lighting system (halogen-Fresnel spotlight), the prototype as well as the optimized simulated V8 light engine indicate that the transitional loss from flood to spot position in halogen-Fresnel spotlight gives a $37 \%$ energy loss, whereas the V8 light engine provides only a 10\% energy loss for the same. The spectrally controlled light output is tunable from 3000 to $6000 \mathrm{~K}$ with dimming capability of a minimum of $5 \%$ of total light intensity of the V8 light engine. The color rendering score $R_{a}>85$ and TLCI $12>70$ indicate that the system is well suited also for studio lighting. Within $\pm 20 \mathrm{deg}$, the intensity is homogeneous with a variation of $11 \%$, whereas the light is projected at $5 \mathrm{~m}$ distance. Similarly, the light output is homogeneous in color within $\pm 23 \mathrm{deg}$, evaluated in $u^{\prime}$ and $v^{\prime}$ (CIE 1976 chromaticity coordinates) with $\Delta u^{\prime} v^{\prime}=0.0010$ while the light is projected at $5 \mathrm{~m}$ distance.

\section{Acknowledgments}

This research was made possible through the project "70710 Højteknologifonden (HTF) V8 Light engine-a revolution for colored light" from Innovation Fund Denmark. The authors would like to acknowledge the technical help from the Danish company Brother, Brother \& Sons ApS, and Thøger Kari. We would also like to thank Maria Louisa Rosenberg Welling from the Technical University of Denmark (DTU) Fotonik for her editorial help.

\section{References}

1. ADB lighting, "'EUROPE' RANGE PC and Fresnel Spotlights," (2000) http://www.adblighting.com/files/DS5113-EEuropePC\&Fresnel datasheet.pdf.

2. Desisti, "LEONARDO 2.000 W Quartz Halogen Fresnel Spotlight" (2012) http://www.desisti.it/cms/ckfinder/userfiles/files/ds/ds_Leonardo_ $2 \mathrm{kw}$.pdf.

3. J. K. Kim and E. F. Schubert, "Transcending the replacement paradigm of solid-state lighting," Opt. Express 16(26), 21835-21842 (2008).

4. A. De Almeida et al., "Solid state lighting review-potential and challenges in Europe," Renew. Sustain. Energy Rev. 34, 30-48 (2014).

5. Y. K. Cheng and K. W. E. Cheng, "General study for using LED to replace traditional lighting devices," in 2nd Int. Conf. on Power Electronics Systems and Applications, ICPESA, pp. 173-177 (2006).

6. J. T. Bailey and P. L. S. Husar, "Multi-color focusable LED light," U.S. Patent No. 005,752,766 A (1998)

7. K. J. Leblanc, L. D. Hagan, and B. Hammond, "LED floodlight system," U.S. Patent No. 2007/0019415 A1 (2007).

8. B. Chemel et al., "Method and apparatus for providing LED-based spotlight illumination in stage lighting applications," U.S. Patent No. 2010/0204841 A1 (2010).

9. E. F. Schubert, Light-Emitting Diodes, 2nd ed., Cambridge University Press, Troy, New York (2006).

10. J. Gadegaard et al., "High-output LED-based light engine for profile lighting fixtures with high color uniformity using freeform reflectors," Appl. Opt. 55(6), 1356-1365 (2016).

11. C.-C. Sun et al., "Collimating lamp with well color mixing of red/ green/blue LEDs," Opt. Express 20(1), A75-A84 (2012).

12. C. C. Sun et al., "Anti-glare LED projection lamp based on an optical design with a confocal double-reflector," Opt. Commun. 285(21-22), 4207-4210 (2012).

13. A. Wilm, "Requirements on LEDs in etendue limited light engines," Proc. SPIE 7001, 70010F (2008).

14. H. Murat, D. Cuypers, and H. De Smet, "Design of new collection systems for multi LED light engines," Proc. SPIE 6196, 619604 (2006).

15. E. Chen and F. Yu, "Design of LED-based reflector-array module for specific illuminance distribution," Opt. Commun. 289, 19-27 (2013).

16. T. Kari et al., "Reliability of point source approximations in compact LED lens designs," Opt. Express 19(Suppl 6), A1190-A1195 (2011).

17. Arri, "L-Series L5-C," (2016), https://www.arri.com/lighting/lighting equipment/lampheads/led_lampheads/1_series/1_series_15_c/.

18. D. Jørgensen, "Color mixing illumination device," U.S. Patent No. 20,140,185,285 A1, pp. 1-15 (2012).

19. J. M. Bornhorst and T. DeSoto, "Additive color mixing system with variable hue and saturation light sources," U.S. Patent No. 5, 031, 078 (1989).

20. S. Gotoh and J. Yao, "Colour mixing method for variable colour lighting and variable color luminaire for use with the method," U.S. Patent No. 005,384,519 A (1995).

21. M. Chakrabarti et al., "Replication of optical microlens array using photoresist coated molds," Opt. Express 24(9), 9528-9540 (2016). 
22. M. Chakrabarti et al., "A color management system for multi-colored LED lighting," Proc. SPIE 9571, 9 (2015).

23. M. Chakrabarti et al., "Monte Carlo analysis of multicolour LED light engine," in CIE, p. OP60 526 (2015).

24. R. Völkel and K. J. Weible, "Laser beam homogenizing: limitations and constraints," Proc. SPIE 7102, 71020J (2008).

25. T. Luo and G. Wang, "Design and optimization for total internal reflection collimators based on slope-error tolerance analysis," Opt. Eng. $\mathbf{5 5}(2), 025103$ (2016).

26. CIE, CIE S 025/E:2015: Test Method for LED Lamps, LED Luminaires and LED Modules (2015).

27. CIE, "Method of measuring and specifying colour rendering properties of light sources," in CIE 13(3), p. 16 (1995)

28. Mike Wood, Television Lighting Consistency Index 2012 (2012).

29. Edmund Optics, Precision Parabolic Mirrors (1992).

Maumita Chakrabarti received her MTech and MS degrees in optoelectronics and in physics from the University of Calcutta in 2000 and Heriot Watt University in 2008, respectively. She obtained her PhD in photonics engineering from the Technical University of Denmark in 2016. She has experience in optical industry as well as in academia for 13 years. Her PhD project was on advance optical design and control of multicolored solid state lighting (SSL) system for stage lighting application.

Henrik Chresten Pedersen is a senior scientist at the Photonics Engineering Department in the Technical University of Denmark. $\mathrm{He}$ has several years of experience with 25 scientific journals and 3 patents.

Paul Michael Petersen is full professor in New Light Sources at the Technical University of Denmark. He has more than 20 years of research experience in lasers, LEDs and optical measuring techniques. From 2002 until 2012, he was appointed as adjunct professor in optics at the Niels Bohr Institute, Copenhagen University. He has authored more than 150 international scientific publications and holds 15 patents.

Christian Poulsen has been working with development of professional stage lighting products since 1999 . He is also working as an external teacher at the Royal Danish School for stagecraft since 1998. His work involves thermal management, control for electronic and embedded software development, optical sensors. He holds a patent on mechanical beam shaping shutters. He also works on flicker-free/highpower switch mode driver electronics and control systems for SSL for the last 12 years.

Peter Behrensdorff Poulsen received his master's degree in materials science from the Technical University of Denmark in 2002 and has worked for more than 15 years within photovoltaics and 10 years within lighting. He provides technical leadership of facility within photovoltaic systems at Department of Photonics Engineering at the Technical University of Denmark. His main research interest is within solar powered lighting systems.

Carsten Dam-Hansen is a senior scientist at the Photonics Engineering Department in the Technical University of Denmark. $\mathrm{He}$ has a MSc, EE and a PhD degrees in laser physics and material interaction, and holographic optical elements for sensors. He has 25 years of experience with 46 scientific journals and 3 patents. He runs a spectro-radiomentric and photometric laboratory and does applied research in LED technology, metrology, and solid state lighting in close collaboration with Danish industry. 